Louisiana State University

LSU Digital Commons

Faculty Publications

Department of Mathematics

$11-1-2014$

\title{
Local stabilization of nonlinear systems through the reduction model approach
}

Frederic Mazenc

CNRS Centre National de la Recherche Scientifique

Michael Malisoff

Louisiana State University

Follow this and additional works at: https://digitalcommons.Isu.edu/mathematics_pubs

\section{Recommended Citation}

Mazenc, F., \& Malisoff, M. (2014). Local stabilization of nonlinear systems through the reduction model approach. IEEE Transactions on Automatic Control, 59 (11), 3033-3039. https://doi.org/10.1109/ TAC.2014.2317292

This Article is brought to you for free and open access by the Department of Mathematics at LSU Digital Commons. It has been accepted for inclusion in Faculty Publications by an authorized administrator of LSU Digital Commons. For more information, please contact ir@lsu.edu. 


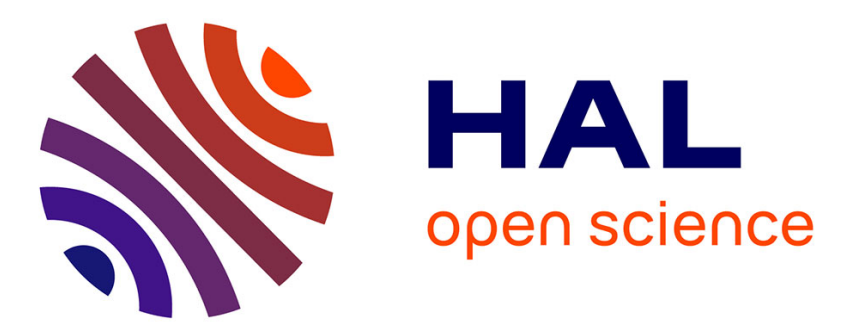

\title{
Local Stabilization of Nonlinear Systems through the Reduction Model Approach
}

\author{
Frédéric Mazenc, Michael Malisoff
}

\section{To cite this version:}

Frédéric Mazenc, Michael Malisoff. Local Stabilization of Nonlinear Systems through the Reduction Model Approach. IEEE Transactions on Automatic Control, Institute of Electrical and Electronics Engineers, 2014, 59 (11), pp.3033-3039. 10.1109/tac.2014.2317292 . hal-01098433

\section{HAL Id: hal-01098433 \\ https://hal.inria.fr/hal-01098433}

Submitted on 14 Jan 2015

HAL is a multi-disciplinary open access archive for the deposit and dissemination of scientific research documents, whether they are published or not. The documents may come from teaching and research institutions in France or abroad, or from public or private research centers.
L'archive ouverte pluridisciplinaire HAL, est destinée au dépôt et à la diffusion de documents scientifiques de niveau recherche, publiés ou non, émanant des établissements d'enseignement et de recherche français ou étrangers, des laboratoires publics ou privés. 


\section{Local Stabilization of Nonlinear Systems Through the 2} 3

\author{
Frederic Mazenc and Michael Malisoff
}

4 Abstract-We study a general class of nonlinear systems with input 5 delays of arbitrary size. We adapt the reduction model approach to prove 6 local asymptotic stability of the closed loop input delayed systems, using 7 feedbacks that may be nonlinear. Our Lyapunov-Krasovskii functionals 8 make it possible to determine estimates of the basins of attraction for the 9 closed loop systems.

10 Index Terms-Delay, nonlinear, reduction model, stabilization.

11

\section{INTRODUCTION}

12 The reduction model approach is a well-known stabilization tech13 nique for systems with input delays. It originated in [1] and has been 14 studied in many works, e.g., [2]-[6]. It is effective for stabilizing 15 linear time-invariant systems with arbitrarily long pointwise or dis16 tributed input delays. However, the approach does not directly apply 17 to nonlinear systems; it is extended by introducing an extra dynamic 18 (which gives the 'state predictor') whose initial condition is given by 19 an implicit equation (as is done in [7]-[9], and [6, Chapt. 6, p. 128]), 20 and only a few recent works adapt it to time varying systems [10]. This 21 is a limitation, because many systems are nonlinear and lead to the 22 stabilization of time varying nonlinear systems when a trajectory has 23 to be tracked. Moreover, the work [11] is limited to globally Lipschitz 24 nonlinear systems, and it has a restriction on the size of the delays. See 25 also [12] and [13] for stabilization of nonlinear systems with arbitrarily 26 long input delays when the systems have special structures, and [14] 27 for compensation of arbitrarily long input delays under input sampling 28 based on prediction.

29 These remarks motivate our work. We show that the reduction 30 model approach can be used to locally asymptotically stabilize a 31 large family of nonlinear time varying systems of the form $\dot{x}(t)=$ $32 A(t) x(t)+B(t) u(t-\tau)+F(t, x(t))$, with arbitrarily long constant 33 known input delays $\tau$, where $F$ is of order 2 in $x$ at the origin. 34 Our key assumption is the stabilizability of a linear approximation of 35 the closed loop system at 0 . Under this assumption, the result seems 36 intuitively obvious. However, to the best of the authors' knowledge, 37 it has never been rigorously established. In particular, the stability 38 of the closed loop system we obtain cannot be proven by applying 39 the Hartman-Grobman theorem, which only applies to ordinary dif40 ferential equations; see [15, Chapt. 1]. One of the crucial benefits 41 offered by our result is that it yields asymptotically stable closed 42 loop systems for which one can determine a suitable subset of the 43 basin of attraction of the closed loop systems. This information is 44 valuable, because it gives a guarantee that some solutions converge to 45 the origin. We estimate the basin of attraction by building a Lyapunov46 Krasovskii functional. It is different from the one in [16], but can be 47 combined with it to establish ISS results. See also [17] for estimates

Manuscript received July 4, 2013; revised December 1, 2013; accepted March 31, 2014. This technical note appeared in part in the American Control Conference, 2014. This work was supported by NSF-ECCS Grant 1102348. Recommended by Associate Editor P. Pepe.

F. Mazenc is with EPI DISCO INRIA-Saclay, L2S, CNRS-Supelec, Gif-surYvette 91192, France (e-mail: frederic.mazenc@1ss.supelec.fr).

M. Malisoff is with the Department of Mathematics, Louisiana State University, Baton Rouge, LA 70803-4918 USA (e-mail: malisoff@1su.edu).

Digital Object Identifier 10.1109/TAC.2014.2317292 of the basins of attraction for time invariant nonlinear systems with 48 predictor feedbacks, under an ISS assumption on the closed loop 49 systems with undelayed controllers. The predictor feedbacks in [17] 50 can be implemented using numerical methods but are totally different 51 from ours, so our work can be viewed as complementary to [17]. 52 Our work is mainly a methodological development, rather than a 53 specific real-world application or experiments. However, input delays 54 naturally arise from measurement and transport phenomena, and our 55 assumptions are very general, so we anticipate that our work can 56 have considerable benefits when applied to mechanical systems where 57 latencies commonly occur.

The rest of this note is organized as follows. We give our definitions 59 in Section II. In Section III, we show how the class of systems we 60 study naturally arises in tracking problems. We state our main result in 61 Section IV, and we prove it in Section V. In Section VI, we discuss a 62 large class of examples where the estimates of the basins of attraction 63 become arbitrarily large when the input delays converge to zero. In 64 Section VII, we illustrate our result in a worked out example. We 65 conclude in Section VIII with a summary of our findings.

\section{Definitions AND Notation}

We let $n \in \mathbb{N}$ be arbitrary and $I_{n}$ denote the identity matrix in 68 $\mathbb{R}^{n \times n}$, and $|\cdot|$ be the usual Euclidean norm of matrices and vectors. 69 For square matrices $M_{1}$ and $M_{2}$ of the same size, we write $M_{1} \geq M_{2} 70$ to mean that $M_{1}-M_{2}$ is nonnegative definite. For each integer $r \geq 1,71$ let $C^{r}$ denote the set of all functions whose partial derivatives up 72 through order $r$ exist and are continuous, and $C^{0}$ denotes the set of all 73 continuous functions, when the domains and ranges are clear from the 74 context. When we want to emphasize the domains and ranges, we use 75 $C^{r}(\mathcal{U}, \mathcal{V})$ to denote the set of all $C^{r}$ functions having domain $\mathcal{U}$ and 76 range $\mathcal{V}$, where $\mathcal{U}$ and $\mathcal{V}$ are suitable subsets of Euclidean spaces. For 77 any constant $\tau \geq 0$ and any continuous function $\varphi:[-\tau, \infty) \rightarrow \mathbb{R}^{n} 78$ and all $t \geq 0$, we define the function $\varphi_{t}$ by $\varphi_{t}(\theta)=\varphi(t+\theta)$ for all 79 $\theta \in[-\tau, 0]$, i.e., the translation operator. Let $\mathcal{K}_{\infty}$ be the set of all $C^{0} 80$ functions $\gamma:[0, \infty) \rightarrow[0, \infty)$ such that $\gamma(0)=0$ and $\gamma$ is strictly 81 increasing and unbounded. Given subsets $S_{1}$ and $S_{2}$ of Euclidean 82 spaces, we say that a function $J: S_{1} \times S_{2} \rightarrow \mathbb{R}^{p}$ is locally Lipschitz 83 with respect to its second argument provided for each compact set $E \subseteq 84$ $S_{2}$, there is a constant $L_{E}$ such that $|J(p, x)-J(p, y)| \leq L_{E}|x-y| 85$ for all $p \in S_{1}$ and all $x \in E$ and $y \in E$. We say that $J$ is strictly in- 86 creasing in its second argument provided the function $Y(x)=J(p, x) 87$ is strictly increasing for each $p \in S_{1}$; we define strictly increasing and 88 nondecreasing in either argument in a similar way. We say that $J$ has 89 order 2 in $y$ at the origin provided there is a continuous function $\alpha$ such 90 that $|J(p, y)| \leq|y|^{2} \alpha(|y|)$ for all $(p, y) \in S_{1} \times S_{2}$. We sometimes 91 omit arguments of functions when the arguments are clear from the 92 context.

\section{Motivation: Tracking Problem}

In this section, we explain how the problem of tracking a trajectory 95 may lead to the problem we solve in the next section. Consider a time 96 varying nonlinear system

$$
\dot{\xi}(t)=g(t, \xi(t))+B(t) \mu(t-\tau)
$$

where the state $\xi$ is valued in $\mathbb{R}^{n}$, the control $\mu$ is valued in $\mathbb{R}^{p}, 98$ $\tau \geq 0$ is a known constant delay, $g=\left(g_{1}, g_{2}, \ldots, g_{n}\right)^{\top}$ is a nonlinear 99 function of class $C^{2}$, and $B$ is a continuous function. The dimensions 100 $n$ and $p$ are arbitrary. We assume that (1) is forward complete for 101 all measurable locally essentially bounded choices for $\mu$, so $\xi(t)$ is 102 
103 defined for all nonnegative times for all such $\mu$ 's. We also assume that 104 there is a nondecreasing function $\gamma$ such that

$$
\max \left\{\left|\frac{\partial^{2}}{\partial \xi^{2}} g_{i}(t, \xi)\right|:|\xi| \leq q, t \geq 0, i \in\{1,2, \ldots, n\}\right\} \leq \gamma(q)
$$

105 for all $q \geq 0$, which exists when $g$ is $C^{2}$ and periodic in $t$.

106 The objective is to follow an admissible trajectory $\xi_{r}$ of class $C^{1}$, 107 meaning the dynamics for $x=\xi-\xi_{r}$ should be asymptotically stable. 108 By admissible, we mean that there is a known continuous function $109 \mu_{r}(t)$ such that $\dot{\xi}_{r}(t)=g\left(t, \xi_{r}(t)\right)+B(t) \mu_{r}(t)$ for all $t \geq 0$. In 110 particular, this means that $\xi_{r}(t)$ is defined for all $t \geq 0$. We assume 111 that $\xi_{r}$ is a known bounded function.

112 Let $x(t)=\xi(t)-\xi_{r}(t)$ and $\mu(t-\tau)=u(t-\tau)+\mu_{r}(t)$. Then 113 the error equation is

$$
\dot{x}(t)=G(t, x(t))+B(t) u(t-\tau)
$$

114 where $G(t, x)=g\left(t, x+\xi_{r}(t)\right)-g\left(t, \xi_{r}(t)\right)$. Notice that $G(t, x)=$ $115 \int_{0}^{1}(\partial g / \partial x)\left(t, \ell x+\xi_{r}(t)\right) x \mathrm{~d} \ell$, so $G(t, x)=(\partial g / \partial x)\left(t, \xi_{r}(t)\right) x+$ $116 F(t, x)$, where

$$
F(t, x)=\int_{0}^{1}\left(\frac{\partial g}{\partial x}\left(t, \ell x+\xi_{r}(t)\right)-\frac{\partial g}{\partial x}\left(t, \xi_{r}(t)\right)\right) x \mathrm{~d} \ell
$$

117 holds for all $t$ and $x$.

118 Applying the Mean Value Theorem and using (2) and the bound119 edness of $\xi_{r}$, we can find a function $\alpha \in C^{0}$ such that $|F(t, x)| \leq$ $120|x|^{2} \alpha(|x|)$. Since $\xi_{r}$ can depend on $t$, the system (3) is time varying, 121 even if $g$ is time-invariant and $B$ is constant. This motivates the study 122 of systems of the form

$$
\dot{x}(t)=A(t) x(t)+B(t) u(t-\tau)+F(t, x(t))
$$

123 where $F$ is of order 2 in $x$ at the origin, which will be our focus for the 124 rest of this note.

\section{Statement of MAIN REsult}

126 We state our main result for (5), where $x$ is valued in $\mathbb{R}^{n}$, the 127 control $u$ is valued in $\mathbb{R}^{p}$ and is to be specified, $\tau \geq 0$ is a given 128 constant delay, and $F$ is a nonlinear function. The dimensions $n$ and $129 p$ are arbitrary. The functions $A, B$ and $F$ are continuous, and $F$ is 130 locally Lipschitz with respect to $x$. The set of all initial conditions we 131 consider is $E_{0}=\left\{\left(\phi_{x}, \phi_{u}\right) \in C^{0}\left([-\tau, 0], \mathbb{R}^{n} \times \mathbb{R}^{p}\right)\right\}$, so the initial 132 times for our trajectories are always 0 . Let $\lambda: \mathbb{R} \times \mathbb{R} \rightarrow \mathbb{R}^{n \times n}$ be 133 the fundamental solution associated with $A$. Then $\lambda\left(t_{0}, t_{0}\right)=I_{n}$ and $134(\partial \lambda / \partial t)\left(t, t_{0}\right)=A(t) \lambda\left(t, t_{0}\right)$ hold for all real numbers $t$ and $t_{0}$. We 135 introduce the following assumptions:

136 Assumption 1:

137 (i) There is a continuous, positive valued, nondecreasing function 138 $h$ such that

$$
|\lambda(t, l) B(l)| \leq h(\tau) \text { for all } t \in \mathbb{R} \text { and } l \in[t, t+\tau] .
$$

139 (ii) There is a constant $a^{+} \geq 0$ such that $\sup _{t \in \mathbb{R}}|A(t)| \leq a^{+}$.

140 Assumption 1 always holds when $B$ is bounded and $A$ is constant, 141 so for instance, it holds for the one-dimensional system

$$
\dot{x}(t)=x(t)+u(t-\tau)+l x^{2}(t) \sin (x(t))
$$

142 where $u \in \mathbb{R}$ is the input, $\tau$ is a positive constant delay, and $l$ is 143 a positive constant. In the case of (7), we can take $A=1, B=1$, $\lambda\left(t, t_{0}\right)=e^{t-t_{0}}$, and $F(t, x)=l x^{2} \sin (x)$, so Assumption 1 holds 144 with $h(\tau)=1$. To ease the readability of our technical assumptions, 145 we will explain how the example (7) satisfies our assumptions, after 146 we introduce each of our three assumptions. Our next assumption is: 147

Assumption 2: There are a continuous function $K:[0, \infty)^{2} \rightarrow 148$ $\mathbb{R}^{p \times n}$, a nondecreasing continuous function $k:[0, \infty) \rightarrow(0, \infty)$, an 149 everywhere positive definite and symmetric function $Q:[0, \infty)^{2} \rightarrow 150$ $\mathbb{R}^{n \times n}$ of class $C^{1}$ with respect to its first argument, and continuous 151 functions $q_{i}:[0, \infty) \rightarrow(0, \infty)$ for $i=1,2,3$ such that $|K(t, \tau)| \leq 152$ $k(\tau)$ for all $(t, \tau) \in[0, \infty)^{2}$, and such that with the choices $H(t, \tau)=153$ $A(t)+\lambda(t, t+\tau) B(t+\tau) K(t, \tau)$ and $R(t, \tau, s)=s^{\top} Q(t, \tau) s$, the 154 following two conditions are satisfied for all $\tau \geq 0$ : (i) Along 155 all trajectories of $\dot{s}(t)=H(t, \tau) s(t)$, we have $\dot{R}(t, \tau, s(t)) \leq 156$ $-q_{1}(\tau) R(t, \tau, s(t))$ and (ii) the bounds

$$
q_{2}(\tau) I_{n} \leq Q(t, \tau) \text { and }|Q(t, \tau)| \leq q_{3}(\tau)
$$

are satisfied for all $t \geq 0$.

Assumption 2 holds for (7) as well. In fact, by choosing $K(t, \tau)=159$ $-2 e^{\tau}$, we obtain $H(t, \tau)=1-e^{-\tau} 2 e^{\tau}=-1$, so Assumption 2 is 160 satisfied with $Q(t, \tau)=1 / 2, q_{1}(\tau)=2, q_{2}(\tau)=q_{3}(\tau)=1 / 2$, and 161 $k(\tau)=2 e^{\tau}$. Finally, we assume:

Assumption 3: There are two continuous functions $f_{1}$ and $f_{2}$ that 163 are locally Lipschitz with respect to their last argument, and continu- 164 ous functions $\alpha_{1}$ and $\alpha_{2}$, such that

$$
\begin{gathered}
F(t, x)=\lambda(t, t+\tau) B(t+\tau) f_{1}(t, \tau, x)+f_{2}(t, x) \text { and } \\
\\
\left|f_{1}(t, \tau, x)\right| \leq|x|^{2} \alpha_{1}\left(\tau,|x|^{2}\right) \text { and } \\
\left|f_{2}(t, x)\right| \leq|x|^{2} \alpha_{2}\left(|x|^{2}\right)
\end{gathered}
$$

for all $t \in \mathbb{R}, \tau \geq 0$, and $x \in \mathbb{R}^{n}$. Also, $\beta_{3}(\tau, m)=m \alpha_{1}\left(\tau, m^{2}\right) 166$ is strictly increasing and unbounded in $m$, and $\beta_{4}(m)=m \alpha_{2}\left(m^{2}\right) 167$ is nondecreasing in $m$. Finally, there are continuous functions $\theta_{1}: 168$ $[0, \infty)^{2} \rightarrow(0, \infty)$ and $\theta_{2}:[0, \infty) \rightarrow(0, \infty)$ such that

$$
\begin{gathered}
\left|\alpha_{1}(\tau, b+c)-\alpha_{1}(\tau, c)\right| \leq b \theta_{1}(\tau, b+c) \\
\left|\alpha_{2}(b+c)-\alpha_{2}(c)\right| \leq b \theta_{2}(b+c)
\end{gathered}
$$

are satisfied for all $b \geq 0$ and $c \geq 0$

To see why (7) satisfies Assumption 3, note that for (7), the fact 171 that $\lambda(t, t+\tau) B$ is invertible implies that one can choose $f_{2}=0$ and 172 $f_{1}(t, \tau, x)=l e^{\tau} x^{2} \sin (x)$. Then we can satisfy Assumption 3 for (7) 173 by taking $\alpha_{1}(\tau, m)=l e^{\tau}$ and $\alpha_{2}(m)=0$ for all $m$ and $\tau$.

Returning to the general system (5), it follows from Assumptions 175 $2-3$ that for any constant $\tau>0$ and:

$$
\alpha_{3}(\tau, m)=\frac{q_{3}(\tau)}{\sqrt{q_{2}(\tau)}} \alpha_{2}(m)+2 a \alpha_{1}(\tau, m),
$$

where $a$ is any constant such that

$$
0<a \leq \frac{q_{1}(\tau) \sqrt{q_{2}(\tau)}}{8 k(\tau)}
$$

there are unique positive values $v_{1}(\tau)$ and $v_{2}(\tau)$ (which also depend 178 on $a$ ) such that

$$
\begin{aligned}
v_{1}(\tau) \alpha_{3}\left(\tau, \frac{4}{q_{2}(\tau)} v_{1}^{2}(\tau)\right) & =\frac{q_{1}(\tau) q_{2}(\tau)}{16} \text { and } \\
v_{2}(\tau) \alpha_{3}\left(\tau, \frac{4 h^{2}(\tau)}{a^{2}} v_{2}^{2}(\tau)\right) & =\frac{a^{2}}{4 \tau h^{2}(\tau)} .
\end{aligned}
$$


180 The existence of unique values $v_{1}(\tau)$ and $v_{2}(\tau)$ follows because $181 \beta_{3}(\tau, m)$ is strictly increasing and unbounded in $m$ and $\beta_{4}(m)$ is 182 nondecreasing, so $m \alpha_{3}\left(\tau, m^{2}\right)$ is strictly increasing and unbounded 183 in $m$. The choice of $\alpha_{3}$ in (13) will become clear when we prove:

184 Theorem 1: Let $\tau>0$ be any constant and Assumptions 1-3 185 hold. Let $a$ be any constant satisfying (14), and set $v(\tau)=$ $186 \min \left\{v_{1}(\tau), v_{2}(\tau)\right\}$ where $v_{1}$ and $v_{2}$ are as above. Then, for each 187 initial function $\left(\phi_{x}, \phi_{u}\right) \in C^{0}\left([-\tau, 0], \mathbb{R}^{n} \times \mathbb{R}^{p}\right)$ satisfying

$$
\begin{array}{r}
\sqrt{q_{3}(\tau)}\left|\phi_{x}(0)+\int_{-\tau}^{0} \lambda(0, m+\tau) B(m+\tau) \phi_{u}(m) \mathrm{d} m\right| \\
+\frac{a}{\tau} \int_{-\tau}^{0}(m+2 \tau)\left|\phi_{u}(m)\right| \mathrm{d} m<v(\tau)
\end{array}
$$

188 the unique solution of (5), in closed loop with

$$
\begin{aligned}
u(t)= & -f_{1}(t, \tau, x(t)) \\
& +K(t, \tau)\left[x(t)+\int_{t-\tau}^{t} \lambda(t, m+\tau) B(m+\tau) u(m) \mathrm{d} m\right]
\end{aligned}
$$

189 converges to 0 as $t \rightarrow \infty$. Moreover, (18) locally asymptotically 190 stabilizes (5) to 0.

191 Remark 1: We comment that our control (18) agrees with the 192 standard predictor controller in the linear time invariant case where $193 f_{1}=f_{2}=0$ and $A$ and $B$ are constant. The extra term $-f_{1}(t, \tau, x(t))$ 194 is used to compensate part of the nonlinearity of the system (5). 195 Assumption 2 is a generalization of the standard assumption that $196(A, B)$ is a stabilizable pair, which is the special case of Assumption 1972 where $\tau=0, A$ and $B$ are constant, and where $K$ and $Q$ can also be 198 taken to be constant. However, we allow the delay $\tau>0$ to be as large 199 as we want. On the other hand, since the $q_{i}$ 's are continuous positive 200 valued functions of the delay, they have positive upper and lower 201 bounds over all $\tau \in\left[0, \tau_{M}\right]$ for any constant $\tau_{M}$. Also, the function $202 k$ from Assumption 2 is nondecreasing in $\tau$. Hence, if we are only 203 concerned with a bounded set $\left[0, \tau_{M}\right]$ of possible values for $\tau$, then we 204 can assume in Assumption 2 that the $q_{i}$ 's and $k$ are all positive con205 stants, by replacing them by the constants $\min \left\{q_{1}(\tau): 0 \leq \tau \leq \tau_{M}\right\}$, $206 \min \left\{q_{2}(\tau): 0 \leq \tau \leq \tau_{M}\right\}, \max \left\{q_{3}(\tau): 0 \leq \tau \leq \tau_{M}\right\}$, and $k\left(\tau_{M}\right)$ 207 without relabeling. These observations will be key to our proof in 208 Section VI that for important special cases, our estimate of the domain 209 of attraction becomes arbitrarily large when $\tau \rightarrow 0^{+}$.

210 Remark 2: Assumptions 1-2 always hold when $A$ and $B$ are con211 stant provided $(A, B)$ is stabilizable. Indeed, in that case $\lambda\left(t, t_{0}\right)=$ $212 e^{\left(t-t_{0}\right) A}$, so the stabilizability of $(A, B)$ is equivalent to the stabiliz213 ability of $(A, \lambda(t, t+\tau) B)$. Also, when the $\alpha_{i}$ 's are $C^{1}$, the existence 214 of functions $\theta_{i}$ satisfying the requirements from Assumption 3 follows 215 from the Mean Value Theorem, since Assumption 2 only requires 216 (11), (12) for nonnegative $b$ 's and $c$ 's. Since $F$ is of order 2 in $x$ 217 at 0 , we can always satisfy Assumption 3 with $f_{1}=0$ and $f_{2}=F$. 218 However, these choices may lead to a conservative estimate of the size 219 of the basin of attraction; see the example in Section VII. Our use 220 of a feedback control with distributed terms is motivated by the facts 221 that $\tau$ is arbitrary and $\dot{\xi}(t)=A(t) \xi(t)$ may be exponentially unstable. 222 In general, the explicit expression for $\lambda$ is unknown, but it can be 223 computed in many important cases. This is the case in particular if $224 A$ is constant or $n=1$. We illustrate Theorem 1 in Section VII.

225 Remark 3: In conjunction with our local asymptotic stability result, 226 we have boundedness of the control from Theorem 1, along all of the 227 closed loop trajectories.

\section{PROOF OF THEOREM 1}

Throughout the proof, we consider any solution of (5) in closed loop 229 with (18) for any initial condition satisfying the requirements (17) of 230 Theorem 1, and any constant delay $\tau \geq 0$.

First Part: New Representation of the System: Let $t_{e}$ be any positive 232 real number or $\infty$ such that the solution is defined over $\left[-\tau, t_{e}\right)$. Such 233 a $t_{e}>0$ exists, because the dynamics (5) grows linearly in $x$ in any 234 bounded open neighborhood of $x(0)$. Later we show that $t_{e}$ can always 235 be taken to be $\infty$ for all of the trajectories we are considering. We 236 introduce the operators

$$
\begin{aligned}
& z(t)=x(t)+\Gamma\left(t, u_{t}\right), \text { where } \\
& \Gamma\left(t, u_{t}\right)=\int_{t-\tau}^{t} \lambda(t, m+\tau) B(m+\tau) u(m) \mathrm{d} m .
\end{aligned}
$$

In all of what follows, we assume that $t \in\left[0, t_{e}\right)$ is arbitrary, unless 238 otherwise noted, and we omit some of the arguments of the time 239 derivatives when they are clear, so $\dot{\Gamma}(t)$ means $(d / d t) \Gamma\left(t, u_{t}\right)$. Then 240 the properties of the fundamental matrix give $\dot{\Gamma}(t)=A(t) \Gamma\left(t, u_{t}\right)+241$ $\lambda(t, t+\tau) B(t+\tau) u(t)-B(t) u(t-\tau)$. Using the formula (5) and 242 our decomposition (9) for $F(t, x)$, we obtain

$\dot{z}(t)=A(t) z(t)+\lambda(t, t+\tau) B(t+\tau)\left[u(t)+f_{1}(t, \tau, x(t))\right]$

$$
+f_{2}(t, x(t)) \text {. }
$$

Also, our feedback (18) satisfies $u(t)=-f_{1}(t, \tau, x(t))+244$ $K(t, \tau) z(t)$. Consequently, in terms of our function $H$ from 245 Assumption 2, (20) becomes

$$
\dot{z}(t)=H(t, \tau) z(t)+f_{2}(t, x(t)) .
$$

Assumption 2 ensures global asymptotic stability of the linearizations 247 $\dot{z}(t)=H(t, \tau) z(t)$ of (21) at 0 . Moreover, the equality

$$
x(t)=z(t)-\int_{t-\tau}^{t} \lambda(t, m+\tau) B(m+\tau) u(m) \mathrm{d} m
$$

is satisfied.

Second Part: Decay Conditions: We study the stability of the closed 250 loop system using its representation as (21) coupled with (22). We 251 introduce the operator

$$
\Xi\left(u_{t}\right)=\frac{1}{\tau} \int_{t-\tau}^{t}(m-t+2 \tau)|u(m)| \mathrm{d} m .
$$

Observe for later use that

$$
\int_{t-\tau}^{t}|u(m)| \mathrm{d} m \leq \Xi\left(u_{t}\right) \leq 2 \int_{t-\tau}^{t}|u(m)| \mathrm{d} m .
$$

Then, for all $t \geq 0$, we have

$$
\dot{\Xi}(t) \leq 2|u(t)|-\frac{1}{\tau} \int_{t-\tau}^{t}|u(m)| \mathrm{d} m .
$$

Also, we can use the upper bound on $f_{1}$ from (10), the bound for 255 $K$ given in Assumption 2 and the formula $u(t)=-f_{1}(t, \tau, x(t))+256$ $K(t, \tau) z(t)$ to get $|u(t)| \leq k(\tau)|z(t)|+|x(t)|^{2} \alpha_{1}\left(\tau,|x(t)|^{2}\right)$. More- 257 over, (8) implies that for all $t \geq 0$ and all $z \in \mathbb{R}^{n}$, we have $q_{2}(\tau)|z|^{2} \leq 258$ 
$259 z^{\top} Q(t, \tau) z$. Taking square roots of both sides of the preceding in260 equality and the dividing by $\sqrt{q_{2}(\tau)}>0$ gives

$$
|z| \leq \frac{1}{\sqrt{q_{2}(\tau)}} \sqrt{R(t, \tau, z)} .
$$

261 Combining the last two estimates with (25) gives

$$
\begin{array}{r}
\dot{\Xi}(t) \leq-\frac{1}{\tau} \int_{t-\tau}^{t}|u(m)| \mathrm{d} m+\frac{2 k(\tau)}{\sqrt{q_{2}(\tau)}} \sqrt{R(t, \tau, z(t))} \\
+2|x(t)|^{2} \alpha_{1}\left(\tau,|x(t)|^{2}\right) .
\end{array}
$$

262 We deduce from Assumptions 2-3 that the time derivative of $R$ 263 along all trajectories of (21) satisfies

$$
\begin{aligned}
\dot{R}(t) & \leq-q_{1}(\tau) R(t, \tau, z(t))+2 z(t)^{\top} Q(t, \tau) f_{2}(t, x(t)) \\
& \leq-q_{1}(\tau) R(t, \tau, z(t))+2|z(t)| q_{3}(\tau)\left|f_{2}(t, x(t))\right| .
\end{aligned}
$$

264 From (26), we deduce that

$$
\begin{aligned}
\dot{R}(t) \leq-q_{1}(\tau) & R(t, \tau, z(t)) \\
& +2 q_{3}(\tau) \frac{\sqrt{R(t, \tau, z(t))}}{\sqrt{q_{2}(\tau)}}|x(t)|^{2} \alpha_{2}\left(|x(t)|^{2}\right) .
\end{aligned}
$$

265 Consider the family of functions $S_{\varepsilon}(t, \tau, z)=\sqrt{R(t, \tau, z)+\varepsilon}-$ $266 \sqrt{\varepsilon}$ parameterized by the constant $\varepsilon \in[0,1)$ and let $S=S_{0}$. Since $R$ 267 is of class $C^{1}$ with respect to $t$ and $z$, it follows that for all $\varepsilon \in(0,1)$, 268 the function $S_{\varepsilon}$ is of class $C^{1}$ with respect to $t$ and $z$, while $S$ is only 269 continuous. Also, (29) and Lemma 1 in the Appendix (applied with the 270 choice $r=R(t, \tau, z)$ ) give

$$
\begin{aligned}
\dot{S}_{\varepsilon}(t) \leq & -q_{1}(\tau) \frac{R(t, \tau, z(t))}{2 \sqrt{R(t, \tau, z(t))+\varepsilon}} \\
& +q_{3}(\tau) \frac{\sqrt{R(t, \tau, z(t))}|x(t)|^{2} \alpha_{2}\left(|x(t)|^{2}\right)}{\sqrt{R(t, \tau, z(t))+\varepsilon} \sqrt{q_{2}(\tau)}} \\
\leq & -\frac{q_{1}(\tau)}{2} S(t, \tau, z(t)) \\
& +\frac{q_{3}(\tau)}{\sqrt{q_{2}(\tau)}}|x(t)|^{2} \alpha_{2}\left(|x(t)|^{2}\right) \\
& +\frac{q_{1}(\tau)}{2} \varepsilon^{\frac{1}{4}}[1+S(t, \tau, z(t))]
\end{aligned}
$$

271 along all trajectories of (21).

272 Third Part: Lyapunov-Krasovskii Functionals: Let us consider the 273 family of functions

$$
V_{\varepsilon}\left(t, z, u_{t}\right)=a \Xi\left(u_{t}\right)+S_{\varepsilon}(t, \tau, z)
$$

274 where the constant $a$ satisfies (14) and we omit the argument $\tau$ in $V_{\epsilon}$ 275 to simplify the notation. Then, (27) and (30) give

$$
\begin{aligned}
\dot{V}_{\varepsilon}(t) \leq & \left(\frac{2 a k(\tau)}{\sqrt{q_{2}(\tau)}}-\frac{q_{1}(\tau)}{2}\right) S(t, \tau, z(t)) \\
& +\frac{q_{3}(\tau)}{\sqrt{q_{2}(\tau)}}|x(t)|^{2} \alpha_{2}\left(|x(t)|^{2}\right)
\end{aligned}
$$

$$
\begin{aligned}
& -\frac{a}{\tau} \int_{t-\tau}^{t}|u(m)| \mathrm{d} m+2 a|x(t)|^{2} \alpha_{1}\left(\tau,|x(t)|^{2}\right) \\
& +\frac{q_{1}(\tau)}{2} \varepsilon^{\frac{1}{4}}[1+S(t, \tau, z(t))] .
\end{aligned}
$$

Since $a$ satisfies (14), we get

$$
\begin{aligned}
\dot{V}_{\varepsilon}(t) \leq- & \frac{q_{1}(\tau)}{4} S(t, \tau, z(t))+|x(t)|^{2} \alpha_{3}\left(\tau,|x(t)|^{2}\right) \\
& -\frac{a}{\tau} \int_{t-\tau}^{t}|u(m)| \mathrm{d} m+\frac{q_{1}(\tau)}{2} \varepsilon^{\frac{1}{4}}[1+S(t, \tau, z(t))]
\end{aligned}
$$

where $\alpha_{3}$ was defined in (13).

Next, we find a suitable upper bound on the term 278 $|x(t)|^{2} \alpha_{3}\left(\tau,|x(t)|^{2}\right)$ from (33). Our formula (22) for $x(t), 279$ Assumption 1, and our bound (26) on $|z|$ give

$$
\begin{aligned}
|x(t)| & \leq|z(t)|+h(\tau) \int_{t-\tau}^{t}|u(m)| \mathrm{d} m \\
& \leq \frac{1}{\sqrt{q_{2}(\tau)}} S(t, \tau, z(t))+h(\tau) \int_{t-\tau}^{t}|u(m)| \mathrm{d} m .
\end{aligned}
$$

Recall that our monotonicity properties of $\beta_{3}$ and $\beta_{4}$ from Assumption 281 3 imply that $m \alpha_{3}\left(\tau, m^{2}\right)$ is strictly increasing as a function of $m 282$ for each $\tau$. Therefore, by separately considering the cases where 283 $S(t, \tau, z(t)) / \sqrt{q_{2}(\tau)} \leq h(\tau) \int_{t-\tau}^{t}|u(m)| \mathrm{d} m$ and where the reverse 284 inequality holds, we get

$$
\begin{aligned}
& |x(t)|^{2} \alpha_{3}\left(\tau,|x(t)|^{2}\right) \\
& \leq \frac{4}{q_{2}(\tau)} S^{2}(t, \tau, z(t)) \alpha_{3}\left(\tau, \frac{4}{q_{2}(\tau)} S^{2}(t, \tau, z(t))\right) \\
& \quad+4 h^{2}(\tau)\left[\int_{t-\tau}^{t}|u(m)| \mathrm{d} m\right]^{2} \alpha_{3}\left(\tau, 4 h^{2}(\tau)\left[\int_{t-\tau}^{t}|u(m)| \mathrm{d} m\right]^{2}\right) .
\end{aligned}
$$

We can combine this inequality with (33) to get

$$
\begin{aligned}
\dot{V}_{\varepsilon}(t) \leq & {\left[-\frac{q_{1}(\tau)}{4}+\frac{4}{q_{2}(\tau)} S(t, \tau, z(t)) \alpha_{3}\right.} \\
& \left.\times\left(\tau, \frac{4}{q_{2}(\tau)} S^{2}(t, \tau, z(t))\right)\right] S(t, \tau, z(t)) \\
+ & {\left[-\frac{a}{\tau}+4 h^{2}(\tau) \int_{t-\tau}^{t}|u(m)| \mathrm{d} m \alpha_{3}\right.} \\
& \left.\times\left(\tau, 4 h^{2}(\tau)\left[\int_{t-\tau}^{t}|u(m)| \mathrm{d} m\right]^{2}\right)\right] \\
& \times \int_{t-\tau}^{t}|u(m)| \mathrm{d} m+\frac{q_{1}(\tau)}{2} \varepsilon^{\frac{1}{4}}[1+S(t, \tau, z(t))] .
\end{aligned}
$$


287 Also, $\quad V_{\varepsilon}\left(t, z(t), u_{t}\right) \geq \sqrt{R(t, \tau, z(t))+\varepsilon}-\sqrt{\varepsilon} \geq S(t, \tau, z(t))-$ $288 \sqrt{\varepsilon}$ and $V_{\varepsilon}\left(t, z(t), u_{t}\right) \geq a \int_{t-\tau}^{t}|u(m)| \mathrm{d} m$ hold for all $\varepsilon \in[0,1)$, by 289 (24). Since $m \alpha_{3}\left(\tau, m^{2}\right)$ is increasing in $m$ for each $\tau$, it follows that:

$$
\begin{aligned}
S(t, & \tau, z(t)) \alpha_{3}\left(\tau, \frac{4}{q_{2}(\tau)} S^{2}(t, \tau, z(t))\right) \\
\leq & {\left[V_{\varepsilon}\left(t, z(t), u_{t}\right)+\sqrt{\varepsilon}\right] \alpha_{3} } \\
& \times\left(\tau, \frac{4}{q_{2}(\tau)}\left[2 V_{\varepsilon}\left(t, z(t), u_{t}\right) \sqrt{\varepsilon}+\varepsilon\right]+\frac{4}{q_{2}(\tau)} V_{\varepsilon}^{2}\left(t, z(t), u_{t}\right)\right) .
\end{aligned}
$$

290 We now apply (11), (12), with $b=\left(4 / q_{2}(\tau)\right)\left(2 V_{\varepsilon}\left(t, z(t), u_{t}\right) \sqrt{\varepsilon}+\right.$ $291 \varepsilon)$ and $c=4 V_{\varepsilon}^{2}\left(t, z(t), u_{t}\right) / q_{2}(\tau)$, and use the fact that $\varepsilon \leq \sqrt{\varepsilon} \leq$ $292 \varepsilon^{1 / 4}$ for all $\varepsilon \in[0,1]$, to find a continuous positive valued and non293 decreasing function $\varphi_{c}$ (also depending on $\tau$, but independent of $\epsilon$ ) 294 such that

$$
\begin{aligned}
\dot{V}_{\varepsilon}(t) \leq[- & \frac{q_{1}(\tau)}{4}+\frac{4}{q_{2}(\tau)} V_{\varepsilon}\left(t, z(t), u_{t}\right) \\
& \left.\times \alpha_{3}\left(\tau, \frac{4}{q_{2}(\tau)} V_{\varepsilon}^{2}\left(t, z(t), u_{t}\right)\right)\right] S(t, \tau, z(t)) \\
+ & \frac{1}{a}\left[-\frac{a^{2}}{\tau}+4 h^{2}(\tau) V_{\varepsilon}\left(t, z(t), u_{t}\right)\right. \\
& \left.\times \alpha_{3}\left(\tau, 4 h^{2}(\tau) \frac{V_{\varepsilon}^{2}\left(t, z(t), u_{t}\right)}{a^{2}}\right)\right] \int_{t-\tau}^{t}|u(m)| \mathrm{d} m \\
& +\varepsilon^{\frac{1}{4}} \varphi_{c}\left(V_{\varepsilon}\left(t, z(t), u_{t}\right)\right) .
\end{aligned}
$$

295 Next, recall that our assumption (17) implies that $\sqrt{q_{3}(\tau)}|z(0)|+$ $296(a / \tau) \int_{-\tau}^{0}(m+2 \tau)|u(m)| \mathrm{d} m<v(\tau)$, where $v(\tau)=\min \left\{v_{1}(\tau)\right.$, $\left.297 v_{2}(\tau)\right\}$ as before. Then (8) from Assumption 2 gives $V_{0}\left(0, z(0), u_{0}\right)<$ $298 v(\tau)$. Since $\sqrt{c_{1}+c_{2}} \leq \sqrt{c_{1}}+\sqrt{c_{2}}$ holds for all nonnegative con299 stants $c_{1}$ and $c_{2}$, we know that $V_{\varepsilon} \leq V_{0}$ holds pointwise for all $300 \varepsilon \in(0,1]$. It follows that $V_{\varepsilon}\left(0, z(0), u_{0}\right) \leq V_{0}\left(0, z(0), u_{0}\right)<\bar{v}$ hold 301 for all $\varepsilon \in(0,1]$, where $\bar{v}=\left[V_{0}\left(0, z(0), u_{0}\right)+v(\tau)\right] / 2>0$. Then $302 \bar{v}<v(\tau)$.

303 Set $\bar{v}_{a}=(v(\tau)+\bar{v}) / 2$. Since $m \alpha_{3}\left(\tau, m^{2}\right)$ is strictly increasing in $304 m$, and since $\bar{v}_{a}<v(\tau)=\min \left\{v_{1}(\tau), v_{2}(\tau)\right\}$, it follows from our 305 conditions (15), (16) on $v_{1}(\tau)$ and $v_{2}(\tau)$ that the constants:

$$
\begin{aligned}
& \bar{p}_{1}=\frac{q_{1}(\tau)}{4}-\frac{4}{q_{2}(\tau)} \bar{v}_{a} \alpha_{3}\left(\tau, \frac{4}{q_{2}(\tau)} \bar{v}_{a}^{2}\right) \text { and } \\
& \bar{p}_{2}=\frac{a^{2}}{\tau}-4 h^{2}(\tau) \bar{v}_{a} \alpha_{3}\left(\tau, 4 h^{2}(\tau) \frac{\bar{v}_{a}^{2}}{a^{2}}\right)
\end{aligned}
$$

306 are positive for all $\tau>0$. Fix any value of $\varepsilon \in(0,1]$ satisfying

$$
\varepsilon \in\left(0,\left(\frac{\min \left\{\bar{p}_{1}, \bar{p}_{2}\right\} \bar{v}}{4 \varphi_{c}\left(\bar{v}_{a}\right) \max \left\{a^{2}, 1\right\}}\right)^{4}\right]
$$

307 where the left endpoint is omitted because we need $\varepsilon>0$. 308 Next, we prove by contradiction that $V_{\varepsilon}\left(t, z(t), u_{t}\right) \leq \bar{v}$ for all $309 t \geq 0$. Assume that this property does not hold. Then, since $310 \bar{v}_{a}>\bar{v}$ and $V_{\varepsilon}\left(0, z(0), u_{0}\right)<\bar{v}$, we can find a $t_{2}>0$ such that $311 V_{\varepsilon}\left(t, z(t), u_{t}\right) \leq \bar{v}_{a}$ for all $t \in\left[0, t_{2}\right]$ and $V_{\varepsilon}\left(t_{2}, z\left(t_{2}\right), u_{t_{2}}\right)>\bar{v}$. Set $312 t_{1}=\inf \left\{t \leq t_{2}: V_{\varepsilon}\left(p, z(p), u_{p}\right) \geq \bar{v}\right.$ for all $\left.p \in\left[t, t_{2}\right]\right\}$. Then, since $313 t \mapsto V_{\varepsilon}\left(t, z(t), u_{t}\right)$ is continuous, we get $V_{\varepsilon}\left(t, z(t), u_{t}\right) \in\left[\bar{v}, \bar{v}_{a}\right]$ for 314 all $t \in\left[t_{1}, t_{2}\right], V_{\varepsilon}\left(t_{1}, z\left(t_{1}\right), u_{t_{1}}\right)=\bar{v}$, and $\dot{V}_{\varepsilon}\left(t_{1}\right) \geq 0$.
By (36) and the fact that $l \alpha_{3}\left(\tau, l^{2}\right)$ is strictly increasing in $l$

$$
\dot{V}_{\varepsilon}(t) \leq-\bar{p}_{1} S(t, \tau, z(t))-\frac{1}{a} \bar{p}_{2} \int_{t-\tau}^{t}|u(m)| \mathrm{d} m+\varepsilon^{\frac{1}{4}} \varphi_{c}\left(\bar{v}_{a}\right)
$$

for all $t \in\left[t_{1}, t_{2}\right]$. It follows from our lower bound on $\Xi$ from (24) 316 that:

$$
\begin{aligned}
\dot{V}_{\varepsilon}(t) & \leq-\frac{1}{2 \max \left\{a^{2}, 1\right\}} \min \left\{\bar{p}_{1}, \bar{p}_{2}\right\} V_{0}\left(t, z(t), u_{t}\right)+\varepsilon^{\frac{1}{4}} \varphi_{c}\left(\bar{v}_{a}\right) \\
& \leq-\frac{1}{2 \max \left\{a^{2}, 1\right\}} \min \left\{\bar{p}_{1}, \bar{p}_{2}\right\} V_{\varepsilon}\left(t, z(t), u_{t}\right)+\varepsilon^{\frac{1}{4}} \varphi_{c}\left(\bar{v}_{a}\right)
\end{aligned}
$$

for all $t \in\left[t_{1}, t_{2}\right]$. Since $V_{\varepsilon}\left(t, z(t), u_{t}\right) \in\left[\bar{v}, \bar{v}_{a}\right]$ for all $t \in\left[t_{1}, t_{2}\right]$, we 318 deduce that

$$
\begin{aligned}
\dot{V}_{\varepsilon}(t) & \leq-\frac{1}{2 \max \left\{a^{2}, 1\right\}} \min \left\{\bar{p}_{1}, \bar{p}_{2}\right\} \bar{v}+\varepsilon^{\frac{1}{4}} \varphi_{c}\left(\bar{v}_{a}\right) \\
& \leq-\frac{\min \left\{\bar{p}_{1}, \bar{p}_{2}\right\}}{4 \max \left\{a^{2}, 1\right\}} \bar{v}<0
\end{aligned}
$$

for all $t \in\left[t_{1}, t_{2}\right]$ when $\varepsilon$ satisfies (38). It follows that $\dot{V}_{\varepsilon}\left(t_{1}\right)<0.320$ This yields a contradiction with the choice of $t_{1}$. Hence, when (38) 321 holds, we get $V_{\varepsilon}\left(t, z(t), u_{t}\right) \leq \bar{v}$ for all $t \geq 0$, which implies that we 322 can choose $t_{e}=\infty$. Also, arguing as we did before, we get

323

$$
\dot{V}_{\varepsilon}(t) \leq-\frac{\min \left\{\bar{p}_{1}, \bar{p}_{2}\right\}}{2 \max \left\{a^{2}, 1\right\}} V_{\varepsilon}\left(t, z(t), u_{t}\right)+\varepsilon^{\frac{1}{4}} \varphi_{c}(\bar{v}) .
$$

for all $t \geq 0$. This gives a value $t_{c}>0$ such that for all $t \geq t_{c}$, we have 324

$$
V_{\varepsilon}\left(t, z(t), u_{t}\right) \leq \frac{4 \varphi_{c}(\bar{v}) \varepsilon^{\frac{1}{4}}}{\min \left\{\bar{p}_{1}, \bar{p}_{2}\right\}} \max \left\{a^{2}, 1\right\}
$$

(since $V_{\varepsilon}$ is nonnegative valued), and therefore also

$$
\begin{aligned}
\Xi\left(u_{t}\right) & \leq \frac{4 \varphi_{c}(\bar{v}) \varepsilon^{\frac{1}{4}}}{a \min \left\{\bar{p}_{1}, \bar{p}_{2}\right\}} \max \left\{a^{2}, 1\right\} \text { and } \\
S_{\varepsilon}(t, \tau, z) & \leq \frac{4 \varphi_{c}(\bar{v}) \varepsilon^{\frac{1}{4}}}{\min \left\{\bar{p}_{1}, \bar{p}_{2}\right\}} \max \left\{a^{2}, 1\right\}
\end{aligned}
$$

Since $S_{\varepsilon}(t, \tau, z)=\sqrt{R(t, \tau, z)+\varepsilon}-\sqrt{\varepsilon} \geq \sqrt{q_{2}(\tau)}|z|-\sqrt{\varepsilon}$ holds 326 for all $t, \tau$, and $z,(24)$ gives

$$
\begin{aligned}
\max \left\{\int_{t-\tau}^{t}|u(m)| \mathrm{d} m, \sqrt{q_{2}(\tau)}|z|\right\} \\
\leq \sqrt{\varepsilon}+\frac{4 \varphi_{c}(\bar{v}) \varepsilon^{\frac{1}{4}}}{\min \{a, 1\} \min \left\{\bar{p}_{1}, \bar{p}_{2}\right\}} \max \left\{a^{2}, 1\right\}
\end{aligned}
$$

for all $t \geq t_{c}$. Set

$$
\Delta=\max \left\{\frac{1}{\sqrt{q_{2}(\tau)}}, 1\right\}\left(1+\frac{4 \varphi_{c}(\bar{v})}{\min \{a, 1\} \min \left\{\bar{p}_{1}, \bar{p}_{2}\right\}} \max \left\{a^{2}, 1\right\}\right) .
$$

Then, since $\varepsilon \in[0,1]$, it follows that for all $t \geq t_{c}$, the inequalities

$$
|z(t)| \leq \Delta \varepsilon^{\frac{1}{4}} \text { and } \int_{t-\tau}^{t}|u(m)| \mathrm{d} m \leq \Delta \varepsilon^{\frac{1}{4}}
$$

are satisfied. Since $\varepsilon$ is arbitrarily small, we deduce that $|z(t)|$ and 330 $\int_{t-\tau}^{t}|u(m)| \mathrm{d} m$ converge to zero when $t \rightarrow \infty$. This and the first 331 inequality in (34) imply that $x(t) \rightarrow 0$ as $t \rightarrow \infty$. Also, by letting 332 $\varepsilon$ depend on the maximum of $V_{0}$ on a suitable neighborhood of the 333 origin, we can prove the local stability part. This proves the theorem. 334 


\section{Arbitrarily Large Domains of Attraction}

336 Theorem 1 applies for all $\tau>0$. On the other hand, consider the 337 special case where $f_{2}=0$ in the decomposition (9) of $F$. Then, 338 setting $\tau=0$ in (9) and in our control (18) produces the uniformly 339 globally asymptotically stable closed loop system $\dot{x}(t)=[A(t)+$ $340 B(t) K(t, 0)] x(t)$ from Assumption 2. This suggests that the domain 341 of attraction should become arbitrarily large as $\tau \rightarrow 0^{+}$when $f_{2}=0$. 342 Our next theorem implies that this is indeed the case. We will assume 343 that the functions $q_{i}$ and $k$ from Assumption 2 are constant, so we 344 omit their arguments $\tau$. This is not restrictive, since now we only need 345 to consider $\tau$ 's on a bounded interval; see Remark 1 .

346 Corollary 1: Let Assumptions 1-3 hold with $f_{2}=0$ and the $q_{i}$ 's 347 and $k$ all constant. Then for each constant $v_{*}>0$, we can find values $348 a \in\left(0, q_{1} \sqrt{q_{2}} /(8 k)\right)$ and $\tau_{M}>0$ (both depending on $v_{*}$ ) such that: 349 For each initial condition $\left(\phi_{x}, \phi_{u}\right) \in C^{0}\left([-\tau, 0], \mathbb{R}^{n} \times \mathbb{R}^{p}\right)$ satisfying

$$
\begin{array}{r}
\sqrt{q_{3}(\tau)}\left|\phi_{x}(0)+\int_{-\tau}^{0} \lambda(0, m+\tau) B(m+\tau) \phi_{u}(m) \mathrm{d} m\right| \\
+\frac{a}{\tau} \int_{-\tau}^{0}(m+2 \tau)\left|\phi_{u}(m)\right| \mathrm{d} m<v_{*}
\end{array}
$$

350 and each constant delay $\tau \in\left(0, \tau_{M}\right)$, the trajectory of (5) in closed 351 loop with (18) converges to 0 as $t \rightarrow \infty$.

352 Proof: We set $\alpha_{2}=0$, so we have $\alpha_{3}=2 a \alpha_{1}$. Then (15), (16) 353 become

$$
\begin{aligned}
v_{1}(\tau) \alpha_{1}\left(\tau, \frac{4}{q_{2}} v_{1}^{2}(\tau)\right) & =\frac{q_{1} q_{2}}{32 a} \text { and } \\
v_{2}(\tau) \alpha_{1}\left(\tau, \frac{4 h^{2}(\tau)}{a^{2}} v_{2}^{2}(\tau)\right) & =\frac{a}{8 \tau h^{2}(\tau)} .
\end{aligned}
$$

354 For each constant $\tau_{M}>0$, Assumption 3 provides a function $\bar{\gamma}$ of 355 class $\mathcal{K}_{\infty}$ such that $m \alpha_{1}\left(\tau, m^{2}\right) \leq \bar{\gamma}(m)$ for all $\tau \in\left[0, \tau_{M}\right]$ and $356 m \geq 0$. Then, replacing $\alpha_{1}\left(\tau, m^{2}\right)$ in (49) by $\bar{\gamma}(m) / m$ gives

$$
\begin{aligned}
\bar{\gamma}\left(\sqrt{\frac{4}{q_{2}}} v_{1}(\tau)\right) & =\frac{q_{1} \sqrt{q_{2}}}{16 a} \text { and } \\
\bar{\gamma}\left(\frac{2 h(\tau)}{a} v_{2}(\tau)\right) & =\frac{1}{4 \tau h(\tau)}
\end{aligned}
$$

357 for all $\tau \in\left(0, \tau_{M}\right)$. Our proof of Theorem 1 shows that the conclusions 358 of that theorem remain true when $v_{1}(\tau)$ and $v_{2}(\tau)$ are defined to be the 359 solutions of (50). Therefore

$$
\begin{aligned}
& v_{1}(\tau)=\frac{\sqrt{q_{2}}}{2} \bar{\gamma}^{-1}\left(\frac{q_{1} \sqrt{q_{2}}}{16 a}\right) \text { and } \\
& v_{2}(\tau)=\frac{a}{2 h(\tau)} \bar{\gamma}^{-1}\left(\frac{1}{4 \tau h(\tau)}\right) .
\end{aligned}
$$

360 Also, when $\tau$ is sufficiently small, the choice

$$
a=\frac{1}{\sqrt{\bar{\gamma}^{-1}\left(\frac{1}{4 \tau h(\tau)}\right)}}
$$

will satisfy our requirements (14) on $a$, because (52) converges to 0361 as $\tau \rightarrow 0^{+}$and because we are now assuming that the $q_{i}$ 's and $k$ are 362 positive constants. Then (51) become

$$
\begin{aligned}
& v_{1}(\tau)=\frac{\sqrt{q_{2}}}{2} \bar{\gamma}^{-1}\left(\frac{q_{1} \sqrt{q_{2}}}{16} \sqrt{\bar{\gamma}^{-1}\left(\frac{1}{4 \tau h(\tau)}\right)}\right) \text { and } \\
& v_{2}(\tau)=\frac{1}{2 h(\tau)} \sqrt{\bar{\gamma}^{-1}\left(\frac{1}{4 \tau h(\tau)}\right) .}
\end{aligned}
$$

Therefore, both $v_{1}(\tau)$ and $v_{2}(\tau)$ converge to $\infty$ when $\tau \rightarrow 0^{+}$. It 364 follows that $v(\tau) \rightarrow \infty$ as $\tau \rightarrow 0^{+}$, so we can satisfy (48) for small 365 enough $\tau>0$ by choosing $\tau$ such that $v(\tau)>v_{*}$. The corollary now 366 follows from Theorem 1 .

\section{ILLUSTRATIVE EXAMPLE}

368

We illustrate Theorem 1 using the 1 dimensional system from (7), 369 which is

$$
\dot{x}(t)=x(t)+u(t-\tau)+l x^{2}(t) \sin (x(t))
$$

where $u \in \mathbb{R}$ is the input, $\tau$ is a positive constant delay, and $l$ is 371 a positive constant. This system is not globally Lipschitz in the 372 state $x$. With the notation of the previous sections, we have $A=1,373$ $B=1, \lambda\left(t, t_{0}\right)=e^{t-t_{0}}$, and $F(t, x)=l x^{2} \sin (x)$. As we saw in 374 Section IV, (54) satisfies our assumptions with $h(\tau)=1, K(t, \tau)=375$ $-2 e^{\tau}, Q(t, \tau)=1 / 2, q_{1}(\tau)=2, q_{2}(\tau)=q_{3}(\tau)=1 / 2, k(\tau)=2 e^{\tau}, 376$ $f_{2}=0, f_{1}(t, \tau, x)=l e^{\tau} x^{2} \sin (x), \alpha_{1}(\tau, m)=l e^{\tau}$ and $\alpha_{2}(m)=0.377$ According to (14), the inequalities $0<a \leq 1 /\left(8 \sqrt{2} e^{\tau}\right)$ have to be 378 satisfied and, by the expression of $\alpha_{3}$ in (13), $\alpha_{3}(\tau, m)=2 a l e^{\tau} . \quad 379$

Choosing

$$
a=\frac{1}{8 \sqrt{2} e^{\tau}}
$$

we can straightforwardly derive an estimate of the basin of attraction 381 from Theorem 1 by using $v=\min \left\{v_{1}, v_{2}\right\}$, where

$$
v_{1}(\tau)=\frac{1}{2 \sqrt{2} l}
$$

and

$$
v_{2}(\tau)=\frac{1}{64 \sqrt{2} \tau e^{2 \tau} l}
$$

which converge to $\infty$ as $l \rightarrow 0$ for each $\tau>0$. On the other hand, 384 when $\tau \in(0,1]$, we can take

$$
a=\frac{\sqrt{\tau}}{8 \sqrt{2} e^{\tau}}
$$

to obtain the values

$$
v_{1}(\tau)=\frac{1}{2 l \sqrt{2 \tau}}
$$

and

$$
v_{2}(\tau)=\frac{1}{64 l e^{2 \tau} \sqrt{2 \tau}}
$$

so $v(\tau)=\min \left\{v_{1}(\tau), v_{2}(\tau)\right\}$ converges to $\infty$ as $l$ converges to zero 388 for fixed $\tau>0$, or as $\tau$ converges to zero for fixed $l$, so the basin 389 of attraction becomes arbitrarily large. This gives convergence of the 390 closed loop solution to 0 . 
392 If, on the other hand, we had chosen, $f_{1}=0$ and $f_{2}(t, x)=$ $393 l x^{2} \sin (x)$, then one could choose $\alpha_{1}=c_{0}$ for any constant $c_{0}>0$ 394 and $\alpha_{2}(m)=l$. This gives $\alpha_{3}(\tau, m)=2 a c_{0}+(1 / \sqrt{2}) l$. Then the 395 corresponding solutions of (15), (16) with the choice

$$
a=\frac{1}{8 \sqrt{2} e^{\tau}}
$$

396 satisfy

$$
v_{1}(\tau) \leq \frac{\sqrt{2}}{16 l}
$$

397 and

$$
v_{2}(\tau) \leq \frac{1}{256 \sqrt{2} e^{2 \tau} \tau l}
$$

398 which would mean that $v(\tau)=\min \left\{v_{1}(\tau), v_{2}(\tau)\right\}$ does not converge 399 to $\infty$ as $\tau$ goes to zero. Thus, the choice $f_{1}=0$ and $f_{2}(t, x)=$ $400 l x^{2} \sin (x)$ is conservative.

\section{$401 \quad$ VIII. CONCLUSION}

402 Stabilization of nonlinear systems with input delays is a central 403 problem that has been studied by many authors using model reduction, 404 prediction, and other methods. Here we adapted the reduction model 405 approach to the problem of locally asymptotically stabilizing the origin 406 of time varying nonlinear systems with pointwise input delays. Our 407 method of proof makes it possible to determine an estimate of the basin 408 of attraction. The result can be adapted to the case where the delay in 409 the input is distributed. Our results can be combined with those of [5] 410 and [10].

411

412

413 We used the following to get (30) in the second part of the proof of 414 Theorem 1:

415 Lemma 1: Let $\varepsilon \in(0,1]$ be a positive real number. Then

$$
-\frac{r}{\sqrt{r+\varepsilon}} \leq-\sqrt{r}+\varepsilon^{\frac{1}{4}}[1+\sqrt{r}]
$$

416 holds for all $r \geq 0$.

417 Proof: Let $r \geq 0$ be given. We first prove that

$$
\frac{r}{\sqrt{r+\varepsilon}} \geq \frac{1}{\sqrt{1+\sqrt{\varepsilon}}} \sqrt{r}-\varepsilon^{\frac{1}{4}} .
$$

418 If $\sqrt{r} /(\sqrt{1+\sqrt{\varepsilon}})-\varepsilon^{1 / 4} \leq 0$, then (65) is satisfied. On the 419 other hand, if $\sqrt{r} /(\sqrt{1+\sqrt{\varepsilon}})-\varepsilon^{1 / 4} \geq 0$, then $r \geq(1+\sqrt{\varepsilon}) \sqrt{\varepsilon}$. 420 It follows that $(\sqrt{\varepsilon}+1) r \geq(1+\sqrt{\varepsilon}) \varepsilon+r \geq \varepsilon+r$. Consequently, $421 r /(r+\varepsilon) \geq 1 /(\sqrt{\varepsilon}+1)$. Taking the square root, and then multiply422 ing through by $\sqrt{r}$, we obtain

$$
r \sqrt{\frac{1}{r+\varepsilon}} \geq \frac{\sqrt{r}}{\sqrt{\sqrt{\varepsilon}+1}} .
$$

Therefore, (65) holds in both cases. Next, observe that (65) implies 423 that

$$
\begin{aligned}
-\frac{r}{\sqrt{r+\varepsilon}} & \leq-\sqrt{r}+\left[1-\frac{1}{\sqrt{1+\sqrt{\varepsilon}}}\right] \sqrt{r}+\varepsilon^{\frac{1}{4}} \\
& \leq-\sqrt{r}+[\sqrt{1+\sqrt{\varepsilon}}-1] \sqrt{r}+\varepsilon^{\frac{1}{4}}
\end{aligned}
$$

Hence, the relation $\sqrt{b+c} \leq \sqrt{b}+\sqrt{c}$ for nonnegative values $b 425$ and $c$ gives $-r / \sqrt{r+\varepsilon} \leq-\sqrt{r}+\varepsilon^{1 / 4} \sqrt{r}+\varepsilon^{1 / 4}$. This gives the 426 conclusion.

\section{REFERENCES}

[1] D. Mayne, "Control of linear systems with time delay," Electron. Lett., 429 vol. 4, no. 20, pp. 439-440, 1968.

[2] Z. Artstein, "Linear systems with delayed controls: A reduction," IEEE 431 Trans. Autom. Control, vol. 27, no. 4, pp. 869-879, 1982.

[3] W. Kwon and A. Pearson, "Feedback stabilization of linear systems 433 with delayed control," IEEE Trans. Autom. Control, vol. 25, no. 2, 434 pp. 266-269, 1980.

[4] A. Manitius and A. Olbrot, "Finite spectrum assignment problem 436 for systems with delays," IEEE Trans. Autom. Control, vol. 24, no. 4, 437 pp. 541-553, 1979.

438

[5] F. Mazenc and D. Normand-Cyrot, "Reduction model approach for 439 linear systems with sampled delayed inputs," IEEE Trans. Autom. Con- 440 trol, vol. 58, no. 5, pp. 1263-1268, 2013.

441

[6] E. Witrant, "Stabilisation des systèmes commandés par réseaux," 442 Ph.D. dissertation, INPG, Laboratoire d'Automatique de Grenoble, 443 Grenoble, France, 2005.

[7] D. Georges, G. Besançon, Z. Benayache, and E. Witrant, "A nonlinear 445 state feedback design for nonlinear systems with input delay," in Proc. 446 Eur. Control Conf., Kos, Greece, 2007, pp. 348-353.

447

[8] M. Krstic, Delay Compensation for Nonlinear, Adaptive, PDE Systems. 448 Boston, MA: Birkhauser, 2009.

[9] M. Krstic and N. Bekiaris-Liberis, "Compensation of infinite-dimensional 450 input dynamics," IFAC Annu. Rev., vol. 34, no. 2, pp. 233-244, 2010.

[10] F. Mazenc, M. Malisoff, and S.-I. Niculescu, "Reduction model approach 452 for linear time-varying systems with delays," IEEE Trans. Autom. Control, 453 to be published.

454

[11] F. Mazenc and S.-I. Niculescu, "Generating positive and stable solutions 455 through delayed state feedback," Automatica, vol. 47, no. 3, pp. 525-533, 456 2011.

[12] M. Krstic, "Input delay compensation for forward complete and feedfor- 458 ward nonlinear systems," IEEE Trans. Autom. Control, vol. 55, no. 2, 459 pp. 287-303, 2010

460

[13] F. Mazenc, S. Mondié, and R. Francisco, "Global asymptotic stabilization 461 of feedforward systems with delay in the input," IEEE Trans. Autom. 462 Control, vol. 49, no. 5, pp. 844-850, 2004.

463

[14] I. Karafyllis and M. Krstic, "Nonlinear stabilization under sampled and 464 delayed measurements, with inputs subject to delay and zero-order hold," 465 IEEE Trans. Autom. Control, vol. 57, no. 5, pp. 1141-1154, 2012.

[15] M. Malisoff and F. Mazenc, Constructions of Strict Lyapunov Func- 467 tions, ser. Communications and Control Engineering Series. New York: 468 Springer, 2009.

[16] F. Mazenc, S.-I. Niculescu, and M. Krstic, "Lyapunov-Krasovskii func- 470 tionals and application to input delay compensation for linear time- 471 invariant systems," Automatica, vol. 48, no. 7, pp. 1317-1323, 2012.

[17] N. Bekiaris-Liberis and M. Krstic, "Compensation of state-dependent 473 input delay for nonlinear systems," IEEE Trans. Autom. Control, vol. 58, 474 no. 2, pp. 275-289, 2013. 
AUTHOR QUERY

NO QUERY. 